Revue

Revue de l'histoire des religions

de Ihistoire des religions

4 | 2013

Sermo mysticus Mystique et langage entre Moyen Âge et époque moderne

\title{
De la liquéfaction à la défaillance : pour un vocabulaire mystique au XII ${ }^{\mathrm{e}}$ siècle
}

From liquefaction to fainting : towards a mystical vocabulary in the twelfth century

Patrice Sicard

(2) OpenEdition

Journals

Édition électronique

URL : http://journals.openedition.org/rhr/8171

DOI : 10.4000/rhr.8171

ISSN : $2105-2573$

Éditeur

Armand Colin

Édition imprimée

Date de publication : 1 décembre 2013

Pagination : 447-483

ISBN : 978-2200928667

ISSN : 0035-1423

Référence électronique

Patrice Sicard, « De la liquéfaction à la défaillance : pour un vocabulaire mystique au x॥ ${ }^{\mathrm{e}}$ siècle », Revue de l'histoire des religions [En ligne], 4 | 2013, mis en ligne le 01 décembre 2016, consulté le 10 décembre 2020. URL : http://journals.openedition.org/rhr/8171 ; DOI : https://doi.org/10.4000/rhr.8171

Tous droits réservés 


\title{
De la liquéfaction à la défaillance : pour un vocabulaire mystique au XII ${ }^{\mathrm{e}}$ siècle
}

\begin{abstract}
La notion de liquefactio apparaît dans le vocabulaire mystique du Moyen Âge central au sein d'une constellation de termes (dilatatio, bullitio, defectio) dont l'utilisation est accréditée dans le lexique mystique par la caution que lui donnent les écoles du XII siècle, celle de Saint-Victor avec ses maîtres, et «l'école du cloître » des milieux cisterciens, comme aussi, secondairement dans la littérature issue des moines noirs. On décrit et circonscrit les accentuations que reçoit en ces deux univers intellectuels, de théoriciens ou de descriptifs, la notion de liquéfaction mystique, dans ses rapports avec l'anthropologie spirituelle (les sens spirituels), l'histoire du salut, la cosmologie (les quatre éléments). On tente de la situer dans le procès mystique médiéval.
\end{abstract}

\section{From liquefaction to fainting: towards a mystical vocabulary} in the twelfth century

The notion of liquefactio appears in the mystical vocabulary of the Middle Ages as part of a constellation of terms (dilatatio, bullitio, defectio) which came into use in the mystical lexicon thanks to twelfth-century schools such as that of Saint Victor with its masters and the "school of the cloister" within the Cistercian world, and also, secondarily, in literature written by the Blackfriars. The notion of mystical liquefaction is studied in these theoretical and descriptive contexts, in its links with spiritual anthropology (the spiritual senses), the history of salvation, and cosmology (the four elements). The notion is ultimately situated within the medieval mystical process. 
«La pollution multiforme des fruits de la terre n'est devenue possible qu'après la liquéfaction des âmes et leur liquidation par les vivants. »

Armel Guerne, lettre à D. Jean-Nesmy

La notion de liquéfaction ${ }^{1}$ fait partie de celles dont la présence, à la fois constante et discrète, dans la littérature spirituelle, donne à suspecter qu'on est devant une notion non pas périphérique au vocabulaire mystique, mais qui touche aux descriptions des traits essentiels de l'expérience mystique ${ }^{2}$. Le terme n'aura pas fait l'objet d'études spécifiques ${ }^{3}$, à l'exception de deux auteurs récents, qui l'abordent indirectement puis directement pour une période donnée ${ }^{4}$. Nous nous proposons ici non pas d'abord d'en suivre, selon un ordre principalement génétique, l'histoire et les avatars,

1. On aura reconnu dans le titre une allusion à l'article d'Hélène Michon, «De l'anéantissement à la liquéfaction», Pour un vocabulaire mystique au XVII siècle. Séminaire du professeur Carlo Ossola (Collège de France), éd. François Trémolières, Turin, Nino Aragno Editore, 2004, p. 243-262.

2. Petit confirmatur: les simples relevés de termes importants dans les éditions critiques de textes médiévaux («tables des mots latins», «table analytique») des années soixante du siècle dernier jusqu'à la première décennie du $\mathrm{XXI}^{\mathrm{e}}$ siècle où les instruments de travail électroniques s'y sont substitués, comportent presque toujours le lemme liquefactio et ses dérivés (liquescens). L'attestation est régulière et les occurrences en faible nombre (Guillaume de Saint-Thierry, De natura et dignitate amoris; Achard de Saint-Victor, Sermons).

3. Ni même d'article de dictionnaire: nulle notice ne lui est consacrée par le Dictionnaire de spiritualité; Jean Leclercq (Otia monastica. Études sur le vocabulaire de la contemplation au moyen âge, Rome, Pontificium institutum S. Anselmi, Herder, [«Studia Anselmiana », 51] , 1963) ne mentionne jamais la notion; une douzaine de renvois dans la somme de Robert Javelet, Image et ressemblance au douzième siècle. De saint Anselme à Alain de Lille, Paris, Letouzey et Ané, 1967, t. 2, p. 360, font référence à quelques auteurs : les commentaires, qui peinent souvent à ordonner les vues, en sont toujours rapides. Au mieux on la rencontre régulièrement dans ces lexiques, précieux mais limités, que parfois les éditeurs adjoignent heureusement aux œuvres éditées: ainsi pour l'édition française de Ruusbroec (Écrits IV, Bégrolles-en-Mauges, abbaye de Bellefontaine, 1999, «Glossaire», p. 375-385, p. 381), ou pour Richard de Saint-Victor et le «Yves» auteur de l'Épître à Séverin sur la charité (éd. Gervais Dumeige, Paris, Vrin, 1955, «Index analytique», p. 192-200, p. 199-200).

4. Indirectement, car unie à deux autres métaphores, dans l'étude fondamentale de Jean Pépin, «Stilla aquae modica multo infusa vino, ferrum ignitum, luce perfusus aer. L'origine de trois comparaisons familières à la théologie mystique médiévale», dans Miscellanea André Combes, t. 1, Rome-Paris, Libreria ed. della 
sur quelque dix-huit siècles ${ }^{5}$, mais plutôt de nous arrêter à quelques moments majeurs et médiévaux de l'histoire de cette notion dans l'expression mystique, ajoutant ainsi une nouvelle période, celle du Moyen Âge central, à celles des précédentes études sur l'histoire du thème qui portaient l'une sur le Moyen Âge tardif, avec Gerson et ses sources, l'autre sur l'âge baroque (François de Sales, Bérulle, Canfeld). La «renaissance du XII ${ }^{\mathrm{e}}$ siècle», pourra, dans son usage du thème, fournir des clefs d'intelligence déterminantes pour son histoire ultérieure.

Le thème de la liquéfaction mystique ne peut invoquer en sa faveur que des attaches scripturaires minimales: de la douzaine d'occurrences bibliques, la littérature mystique s'est préférentiellement attardée à deux ou trois, empruntées à la littérature sapientielle, et dont elle s'est attachée à épuiser les virtualités de sens : «mon âme s'est liquéfiée à la voix du Bien-aimé» du Cantique (V, 6) (anima mea liquefacta est ut locutus est), le psaume XXI, 15 : factum est cor meum tanquam cera liquescens («mon cœur est devenu comme une cire liquide») et, plus rarement et selon la lecture messianique: Emittet Verbum suum et liquefaciet ea (Ps. 147, 18, «il enverra son Verbe et il les rendra liquides»). Restera également de ces versets, une comparaison qui reparaîtra régulièrement, voire qui deviendra élément d'argumentation: sicut cera. Quant au sujet de la liquéfaction, lui aussi est simplement repris: le cœur ou l'âme (cor, anima), sans préjudice, à partir surtout du XII ${ }^{\mathrm{e}}$ siècle et de sa psychologie affinée, de précisions à l'intérieur de ce cœur ou de cette âme, quant aux facultés ou instances intéressées ou à celles qui interviennent, fût-ce antérieurement et comme condition.

Comme en témoigne le texte mis en exergue, «liquéfaction» est pris en mauvaise part dans le monde moderne. Il n'a pas toujours eu, dans le monde antique puis médiéval, ces connotations négatives. Dans le langage traditionnel de la mystique, il est souverainement positif, encore qu'il puisse, chez ceux-là mêmes qui en connaissent le rôle dans la traduction de l'expérience spirituelle, recevoir une

Pont. università lateranense-Vrin, 1967, p. 331-375. S'ajoute dernièrement l'étude d'Hélène Michon (v. n. 1), qui se circonscrit à la période baroque.

5. Des attaches sont signalées par J. Pépin, art. cit., et par R. Javelet, op. cit., t. 2, p. 25, n. 193: pour «l'image du fer plongé dans le feu et transfiguré en lui», avec renvoi à Maxime (Ambigua 2, PL 122, col. 1202B), Basile (Contra Eunomium III, 2, PG 77, 788 A); Cyrille de Jérusalem (Cathéchèses mystagogiques 17, 14). 
connotation négative. Ces ambivalences recoupent, pour une part, deux des lieux scripturaires générateurs: le ps. 21, 15 (Factum est cor meum tanquam cera liquescens in medio ventris mei) est aisément inducteur d'une perception défavorable: le cœur est au centre, mais s'il se laisse «fondre dans une molle dégénérescence» (quadam mollitie liquescens) par la concupiscence charnelle, alors totum defluxit in ventrem et in medium ventris («il est passé tout entier jusque dans le ventre et au milieu du ventre») et «l'affection naturelle de l'amour » est changée «en un brutal appétit de la chair $»^{6}$. En ce contexte, la liquefactio est effet des puissances affectives et d'une des formes perverses de l'amour qu'est la convoitise ou cupiditas, par opposition à la charité. Ce qui laisse supposer que, chez les augustiniens du XII ${ }^{\mathrm{e}}$ siècle, la liquefactio «positive» sera aussi l'effet de l'amour, sous la forme de la caritas.

Le mouvement mystique du XII ${ }^{\mathrm{e}}$ siècle s'est fait sentir, institutionnellement en quelques lieux qui ont pu en offrir une expression littéraire des expériences ou une systématisation, dans le cadre de la théologie monastique ou de la théologie canoniale. Nous nous arrêterons donc aux mondes victorin et cistercien en particulier et, pour quelque incursion, à celui des moines noirs. Deux auteurs (Hugues de Saint-Victor et saint Bernard), par l'importance qu'ils confèrent à notre thème ou celle de leurs vues, demanderont une esquisse de monographie pour eux seuls, alors que les données, nuances et compléments d'autres (Guillaume de Saint-Thierry, Pierre de Celle) pourront se retrouver sous des têtes de section communes.

\section{LA LIQUÉFACTION DANS LA MYSTIQUE HUGONIENNE}

Les occurrences chez Hugues de Saint-Victor de la liquefactio et des mots de même famille sont peu nombreuses (quelque vingt emplois en contexte spirituel dans tout l'œuvre du victorin). Mais il est à noter que le terme apparaitt toujours en des passages prégnants, qui ont plusieurs fois et pour d'autres raisons retenu l'attention des commentateurs, et que l'on considère par ailleurs comme des pièces d'anthologie.

6. Guillaume de Saint-Thierry, De natura et dignitate amoris 3, 7-17, éd. Marie-Madeleine Davy, Paris, Vrin, 1953, p. 75. 


\section{Liquéfaction et mystique sponsale}

En premier lieu dans le De archa Noe, œuvre centrale par sa date de composition, par sa richesse de contenu, par les méthodes pédagogiques nouvelles mises en œuvre, par ses attaches littéraires enfin avec d'autres œuvres de Hugues?

\section{L'épouse et le treillis : fuite et suite}

L'union ou l'unition étant le fonds commun des états mystiques, et la liquéfaction étant une des composantes de ces états, on peut s'attendre à la retrouver employée dans la mystique sponsale.

Quia ergo homo auditum intus perdidit quo Deum loquentem audiat, revocans nos Deus ad se foris clamat. Sed cum loquitur semper quasi occultari volens se subtrahit, ut mentem humanam et per hoc quod loquitur de se admoneat, et per hoc quod se occultando fugit ad se trahat. Irritat enim desiderium nostrum, ut augeat, quia et loquendo amorem suum in nobis excitat et fugiendo, ut se sequamur, inflammat. Tale est enim cor hominis, ut si quod diligit adipisci non valeat, amplius desiderio suo inardescat. Sic in Canticis canticorum sponsus venit, stat post parietem, prospicit per fenestras et cancellos, ut quasi lateat et non lateat. Mittit manum suam per foramen, tangit sponsam et sono depresso, voce tenui vocat eam, et dicit: "Veni amica mea, speciosa mea, columba mea. Ecce hiemps transiit, imber abiit, et recessit. Flores apparuerunt in terra nostra; vox turturis audita est in terra nostra". Illa ut audit Sponsum adesse surgit mox, festinat, aperit pessulum hostii sui et quasi venientem exceptura brachia ad amplectendum parat. Vix tolerat, vix sustinet, vix exspectat. Liquescit anima ejus, ardet cor ejus, fervent praecordia ejus, exsultat, gaudet, tripudiat, jucundatur, ruit obviam venienti ${ }^{8}$.

7. À savoir le De tribus diebus, le Libellus de formatione arche, le De vanitate rerum mundanarum et le Dialogus de creatione mundi qui y sont annoncés ou amorcés ou utilisés.

8. Hugues de Saint-Victor, De archa Noe, éd. Patrice Sicard, Turnhout, Brepols, («Hugonis de Sancto Victore opera», 1, «Corpus Christianorum Continuatio Mediaevalis», 176), 2001, IV, iv, 25-44. «L'homme ayant perdu au-dedans l'oreille par laquelle il pouvait écouter Dieu, Dieu nous rappelle à lui et crie au-dehors. Mais lorsqu'il parle, il se soustrait toujours, comme désireux de se dissimuler, afin d'avertir l'esprit humain de sa présence par ce qu'il dit, et de l'attirer à lui, parce qu'il fuit en se cachant. Il irrite donc notre désir pour l'augmenter, car, en parlant, il excite en nous son amour, et, en fuyant, il nous embrase du désir de le suivre. Tel est le cœur de l'homme: s'il ne peut atteindre ce qu'il aime, il brûle d'un plus ardent désir. Ainsi, dans le Cantique des cantiques, le fiancé vient, il se cache derrière la muraille et regarde par la fenêtre à travers le treillis, comme pour se cacher et ne pas se cacher. Il passe sa main par l'ouverture, touche l'épouse, et, très doucement, d'une voix faible, il l'appelle et dit: Viens 
Liquescit anima eius: l'expression paraît en compagnie de termes techniques de la langue mystique médiévale, tripudatio et iubilus.

Il importe de remarquer d'abord que l'acte à l'origine de la liquefactio est une initiative divine et qu'il consiste en un éveil de deux sens spirituels, hiérarchisés et successifs. Vient en premier l'ouïe qui perçoit la voix du Bien-aimé. Elle implique encore quelque distance, qui tend à diminuer. S'y joint le toucher, sens spirituel fondamental, et qui ici prend la forme minimale d'un effleurement du bout des doigts dont la suite laisse entendre qu'il a pour fonction d'éveiller par sa fugacité même le désir de l'embrassement.

Il faut noter en second lieu que cette rencontre interpersonnelle introduit une phase d'intermittences du cœur et de la présence sentie, d'allers et retours, thème fréquent sous la plume du victorin comme dans la littérature spirituelle ${ }^{9}$. Ces dérobades pédagogiques ont pour effet d'aviver le désir qu'a fait naître l'effleurement pour attirer à la suite de l'Époux.

C'est là semble-t-il qu'intervient la liquefactio dont un des effets sera précisément de mettre en état de le suivre. Cette sequela est une forme inchoative du «passage» en Dieu qui est en ce texte l'entrée dans le locus amoenus. Celui-ci a une double dimension, pouvant s'agir du jardin eschatologique ou de ses commencements que serait le jardin de l'expérience mystique, paradis retrouvé. Mais l'insistance est ici sur la dimension eschatologique:

Sed ille, cum jam teneri putatur, declinat et subito quasi de mediis elapsus amplexibus fugit. Quid est hoc? Dum non quaeritur, quaerit, dum non vocatur, venit, dum autem quaeritur, declinat, et dum vocatur, fugit. Si non diligit, quare venit, aut si diligit, quare fugit? Diligit autem et ideo venit, sed quia hic non diligit, ideo fugit. Quid est hoc, quod dixi, hic non diligit? In hoc mundo, in hoc saeculo, in hac terra, in hac patria, in hoc exsilio. Sed vocat nos ad terram suam, ad patriam suam, quia non decet talis amor patriam istam. Immunitio amoris esset vilitas regionis. Iocundus amor amenum querit locum, ideo commendat nobis terram suam, ideo laudat patriam suam, ideo dicit: "Flores apparuerunt

mon amie, mon épouse, ma colombe. Voici que l'hiver s'en est allé, les pluies se sont dissipées et ont cessé. Les fleurs ont apparu sur notre terre; la voix de la tourterelle s'est fait entendre sur notre terre. Celle-ci, dès qu'elle entend l'Époux, se lève aussitôt, elle se hâte, tire le verrou de la porte et étend les bras comme pour embrasser celui qui vient. Elle n'y peut plus tenir; ne peut plus supporter; ne peut plus attendre, son âme se fond en elle, son cœur brûle, sa poitrine est en feu; elle exulte, se réjouit, trépigne, jubile, s'élance au-devant du visiteur». Sauf mention contraire, toutes les traductions sont de nous.

9. Voir infra n. 17. 
in terra nostra; vinee florentes odorem dederunt; vox turturis audita est in terra nostra", ut concupiscamus talem regionem, ut desideremus talem patriam, et sequamur eum. Ibi nos amat, ibi amore nostro frui desiderat, ibi amplecti a nobis postulat ${ }^{10}$.

Toutefois ce passage en Dieu n'est pas un écoulement: l'âme, effleurée et liquéfiée, «s'élance» au-devant du visiteur: ce qui implique non tant un épanchement auquel elle s'abandonnerait qu'une activité dont elle serait encore maîtresse au moins pour une part, la consistance d'une décision aboutissant à une action mais sous attraction.

La dimension morale enfin n'est présente que très indirectement en ce texte où la liquéfaction n'est pas la conséquence d'une ardeur ayant d'abord pour but de purifier les scories d'une conduite morale répréhensible ou imparfaite, mais une suite de l'intensité d'un amour corrélatif à la proximité d'une présence. Le fruit de pédagogie spirituelle mentionné est la rééducation de l'espérance et de l'attente qui doit tendre à la possession complète in patria, non à une jouissance totale immédiate. La consistance personnelle est laissée intacte par cette liquéfaction nuptiale; il en sera pareillement pour les autres formes que mentionne le victorin.

\section{La liquéfaction et la fuite: Adam ubi es?}

L'effet proprement moral de la liquefactio est mis nettement en valeur par un passage fameux de l'Homélie VIII sur l'Ecclesiaste ${ }^{11}$.

10. De archa Noe, éd. cit., IV, iv, 44-60. «Mais lui, quand elle pense le tenir, se dérobe, comme s'arrachant soudain aux embrassements, il fuit. Qu'est-ce donc? Il cherche lorsqu' on ne le recherche pas; s'approche quand on ne l'appelle pas; se dérobe quand on le cherche, et fuit quand on l'appelle. Pourquoi vient-il s'il n'aime pas? Et, s'il aime, pourquoi s'enfuit-il? Il aime et donc il vient; mais il s'enfuit, parce que ce n'est pas ici qu'il aime. Qu'est-ce à dire cela: “ce n'est pas ici qu'il aime" ? C'est-à-dire dans ce monde, dans ce siècle, dans cette terre, dans cette patrie, dans cet exil. Mais il nous invite dans la terre qui est sienne, dans la patrie qui est sienne; un tel amour ne convient pas à cette patrie-ci. La bassesse de ces contrées diminuerait l'amour. L'amour joyeux demande un domaine agréable. C'est pourquoi il fait valoir à nos yeux sa patrie, il en chante les louanges, il dit: Les fleurs ont apparu sur notre terre; les vignes en fleurs ont répandu leur odeur, la voix de la tourterelle s'est fait entendre sur notre terre: et cela, pour nous faire désirer cette contrée, soupirer après cette patrie et marcher à sa suite. C'est là qu'il nous aime, là qu'il désire jouir de notre amour, là qu'il demande nos embrassements ».

11. PL 175, col. 164C-169D. Nous conservons, pour faciliter provisoirement renvois et citations, ce titre apocryphe donné à ce qu'il faut regarder comme une 
La liquéfaction remédie à la dureté et à l'endurcissement du cœur (dure, indurate et obdurate); elle est effet de l'amour (tanta benignitas, tanta flamma, tam ingens ardor dilectionis). Une nouvelle faculté de l'âme intervient, la memoria. Elle sert de medium et d'instrument à l'amour dont elle conserve le souvenir (dilectionis memor), lui permettant d'adoucir (emolire) le cœur induré à tel point qu'il en amène la liquéfaction, laquelle introduit dans une course qui en est l'effet noté (ut liquefieres et curreres post eam). En ce contexte, la voix de Dieu au jardin de la Genèse (Adam ubi es ?), et non plus de l'Époux du Cantique tend à éveiller la mémoire et son souvenir, et indirectement à l'amollissement de l'âme. Le sens spirituel (de l'ouie) n'est plus ici cause directe et initiale de la liquéfaction, à la différence du texte précédent: s'il arrivait qu'il soit éveillé par la parole divine qui retentit intus, c'est à son tour qu'il serait principe de réveil du souvenir de la dilection et, par là, de l'amollissement du cœur. L'activité du passage en Dieu est ici également soulignée, car il s'agit d'une sequela qui est une course (avec souvenir probable du Cantique des cantiques I, 3: post te curremus). Cette course n'est pas celle-là seule d'Adam, mais aussi de Dieu, et dans les deux cas elle prend la forme d'allées et venues: de la part de Dieu qui s'éloigne sans s'éloigner mais

Expositio in Ecclesiasten. Il permet l'usage, trompeur mais commode, du terme «homélie» pour renvoyer aux dix-neuf chapitres ou sections ou portions que comporte cette œuvre, inachevée. Nous transcrivons les principaux passages de l'Homélie ici retenus (166A-167D); nous soulignons: «Ubi es Adam, ubi es? Audivi, inquit, vocem tuam et abscondi me. O abscondite, cui abscondisti te? O abscondite, ubi es? [...] Et tu ubi es? Illa intus est, et tu foris es; et ideo clamat tibi, et dicit: Adam ubi es? Quaeris ut tuam ignorantiam arguat; ambulat ut instabilitatem tuam ostendat. Cum deambularet Dominus in paradiso ad horam post meridiem vocavit et dixit: Adam, ubi es? Non ambulavit, sed deambulavit. Quid est deambulavit? Huc et illuc quasi errabundus et vagus in directum non vadens; sed pergens quocunque, hoc est, deambulavit. Et quare ita deambulare voluit? Ut talem se ostenderet foris, qualis intus esse coeperat. Jam enim mota erat veritas, et fluctuabat ut recederet a corde peccatoris. Imo veritas stabat, et peccatrix conscientia fluctuabat; et ideo veritas foris deambulabat, quia intus peccator a veritate fluctuabat. Deambulabat tamen et non discedebat, neque abiit in directum elongans quasi irrevocabilis, nec reversura amplius; sed prope gyrans et juxta deambulans. [...] Idcirco non cito abiisse, sed praestolari adhuc: ituram tamen, et jam, quia non est qui revocet, ituram, et ecce ambulare ut abeat; quoniam nemo est dilectionis memor, qui charitatis recordetur, cui cordi sit societatis affectus. $\mathrm{O}$ infelix Adam, ubi est recordatio tua? Ubi fixisti animam tuam ut haec sustineas? Dure, indurate, et obdurate, non te emollire potuit tanta benignitas, tanta flamma, tam ingens ardor dilectionis, ut liquefieres et curreres post eam?» 
pour rappeler le souvenir de sa présence, de la part de l'homme qui revient ad eum a quo factus est ou, au contraire, se perd dans une errance désormais sans but, vagus et profugus super terram. Pas plus que dans le précédent passage cette course, de Dieu ou d'Adam, n'est un écoulement, s'il reste vrai que la consécution est explicitement et fortement marquée (ut liquefieres et curreres). Mais comme dans le précédent passage également, les alternances de présence et d'absence, de rapprochements et d'éloignements rythment ces démarches. Enfin, un trait net chez le victorin, la dimension cognoscitive est ici encore nettement affirmée sans préjudice de l'amour: c'est la Vérité qui cherche celui qui fuit la vérité sur lui-même, en se cachant dans les obscurités des feuillages.

De l'Adam post-lapsaire à l'Épouse du Cantique, Hugues insère dans les épisodes de l'histoire du salut le terme et la notion de liquefactio: signe d'abord d'une identité substantielle des trois économies successives et concomitantes (ante legem, sub lege, sub gratia) dont la scansion rythme la théologie hugonienne; signe également que cet élément relève pour le victorin non de ce qui sera plus tard appelé les épiphénomènes de la mystique, mais d'une de ses constantes, sinon de ses traits essentiels.

\section{Une variante du fer en fusion : liquidités monétaires}

Dans l'appendice obligé au De archa Noe qu'est le Libellus de formatione arche, la dimension historique est plus forte encore, faisant l'objet d'une systématisation: c'est dans une histoire et un itinéraire spirituel personnel qu'est inséré le phénomène de la liquéfaction, qu'on a vu à l'œuvre dans les épisodes de l'histoire du salut.

Le De archa transmet le contenu d'entretiens tenus au cloître de Saint-Victor dans les années 1125-1126; ils s'étaient accompagnés d'une représentation figurée, sorte de psychocosmogramme géant, où dans la structure de l'arche de Noé, sont insérés et figurés les moments de l'histoire du salut - personnel autant que communautaire - et les étapes de la chute et du relèvement. Cette maquette à trois dimensions, orientée, dont le Libellus décrit les modalités de réalisation, abrite également des figurines, personnalisation des états intérieurs, et qui sont disposées sur quatre montées, de trois paliers 
chacune, et partant des quatre coins du diagramme pour converger vers son centre. Ce sont les montées de l'ascension restauratrice. Une série doit retenir l'attention, celle par laquelle une des suites de la déchéance originelle, l'ignorance, est guérie:

A frigore occidentis iuxta pedem prime scale quidam de caverna obvoluta facie prodiens corruit, et in lapidem offendens vas quod portat confringit ad exprimendam ignorantiam que per varios errores animae integritatem dissipat. Post hec in eadem scala cognitio erecta pingitur contra volumen, quod deorsum extenta manu porrigitur, et iuxta eam fragmenta vasis. In hoc volumine, sicut diximus, scriptum est: In principio fecit Deus coelum et terram, quia primo cognitio Dei in creaturis est. In secunda scala meditatio sedens exprimitur et colligens fragmenta vasis fracti. In tertia scala contemplatio formatur ad similitudinem fabri conflans eadem fragmenta, ita ut liquefactio per ductum coloris, quem superius juxta versum hujus scale sursum porreximus, quasi per fistulam in medium cubitum, quasi in monetam currere videatur. Propter hoc mysterium: quia integritatem animae, quam ignorantia frangit, cognitio invenit, meditatio colligit, contemplatio per ignem divini amoris liquefaciendo in monetam divinae similitudinis reformandam fundit ${ }^{12}$.

Cette image du fer en fusion offrait plusieurs avantages dont deux, compte tenu de la théologie et de la mystique du victorin, ne devaient pas le laisser insensible. C'est d'abord la coextensivité

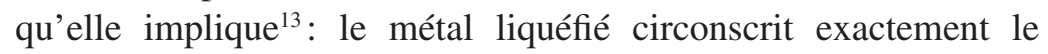
feu divin qui le liquéfie et ce dernier applique son action à ce premier. Elle permet de sauvegarder en mystique une des lois de

12. Hugues de Saint-Victor, Libellus de formatione arche, éd. cit. (voir n. 8), $\mathrm{V}, 23-40$. «Du froid de l'occident, à côté de la base du premier escalier, quelqu'un sort d'une caverne, le visage voilé, et tombe; heurtant une pierre, il brise le vase qu'il portait: ainsi est rendue l'ignorance, dont les diverses erreurs dissolvent l'intégrité de l'âme. Ensuite, sur ce même escalier, la connaissance est peinte debout contre le livre qu'une main tendue tient au-dehors; près d'elle, les débris du vase. Comme on l'a dit, ce livre porte écrit: Au commencement Dieu fit le ciel et la terre, car la première connaissance de Dieu se prend des créatures. Dans le second escalier, la méditation est figurée par une personne assise, qui rassemble les débris du vase brisé. Dans le troisième escalier, la contemplation est représentée sous l'image d'un ouvrier qui fait fondre ces mêmes débris, en sorte que, par ce trait de couleur que nous avons tiré près du verset de cet escalier, cette matière en fusion semble courir comme par un conduit vers le milieu de la coudée, dans ce qui serait un moule. Et en voici le sens : l'intégrité de l'âme que l'ignorance a brisée, la connaissance la retrouve, la méditation la réunit, et la contemplation, la portant en fusion par le feu de l'amour divin, la coule dans le moule de la ressemblance divine».

13. «Comme l'air totalement pénétré de lumière et le fer totalement circonscrit par le feu» (J. Pépin, art. cit., p. 343, à propos d'autres auteurs). 
l'anthropologie spirituelle hugonienne, elle-même impliquée par une ecclésiologie: la rigoureuse coextensivité de la grâce et de l'Église, de l'âme et du corps de celle-ci, tout comme de l'âme et du corps humain. Cette coextensivité se vérifie dès les premiers jours de l'opus restaurationis, puisque la grâce, déjà opérante sous l'économie de la loi de nature puis sous l'économie de la loi mosaïque, y constituait la Cité de Dieu et, du même mouvement, la Cité de l'âme. Cette inclusion réciproque de l'âme en l'Église et de l'Église en l'âme requiert qu'en toutes deux la coextensivité se vérifie également: «il n'est rien hors du corps qui soit mort, rien dans le corps qui ne soit vivant»; pareillement, rien hors de l'application du feu divin qui ne soit en fusion, et nulle liquéfaction qui se puisse concevoir hors lui ${ }^{14}$.

La seconde connotation est moins soulignée par le maître de Saint-Victor qu'elle ne le sera deux siècles plus tard par Gerson. L'image de la goutte d'eau qui se perd dans l'océan ou de la goutte de vin dans le vase d'eau, outre son usage en christologie et en théologie sacramentaire pour l'eucharistie ${ }^{15}$, a pu servir aux mystiques essentielles. Elle a pourtant essuyé, tardivement, des critiques dont Gerson est un représentant: il veut éviter «qu'on dise que l'âme unie à Dieu se transforme en lui de la même façon qu'une goutte d'eau [...] perd son être propre $»^{16}$. Ce souci ne paraît pas avoir été majeur chez Hugues. Il mentionne à plusieurs reprises la permanence de «l'être propre» (le terme substantia est alors souvent utilisé), mais sans insistance particulière. Il reste que l'image de la liquéfaction d'un métal qui change d'aspect et de structure extérieure sans changer de nature pouvait, mieux que la métaphore de la goutte d'eau, préserver une requête de la mystique nuptiale et de l'union des amants: la permanence des identités qui se rencontrent, et qui précisément s'affirment dans ces allers

14. Voir Patrice Sicard, Hugues de Saint-Victor et son école, Turnhout, Brepols, 1991, p. 112-115.

15. Ce qui vaut aussi pour le thème du métal en fusion. Pour celui-ci on pourrait ajouter son usage en eschatologie: il sert au chanoine régulier Gerhoh de Reichesberg pour exposer la transfiguration des fidèles dans l'unité de la Jérusalem céleste: de même qu'un métal liquéfié au feu perd la forme qu'il avait auparavant, tout en conservant sa nature, et revêt celle du feu même, ainsi dans la Jérusalem céleste, les fidèles enflammés par l'amour qu'est l'Esprit saint, ne peuvent être dissemblables de Dieu (In Ps., PL 194, col. 359D-350A, cité par R. Javelet, op. cit., t. 1, p. 359).

16. J. Pépin, art. cit., p. 331-332. 
et retours de présence, depuis un marivaudage spirituel jusqu'aux joutes d'amour auxquelles introduit l'expérience mystique ${ }^{17}$.

\section{Métal en fusion et fragments de poterie à la cuisson}

Cet avantage doctrinal que présente l'image d'une monnaie métallique en fusion n'a pas empêché qu'Hugues ne veuille la doubler en ce même endroit, d'une autre image, celle du vase d'argile. Celle-ci pouvait offrir à son tour d'autres avantages.

Il semble bien que l'esprit de Hugues ait alors été occupé comme par deux arrière-fonds doctrinaux qui s'accompagnaient de deux images à première vue non compossibles: le thème de la glaise du sol dont l'homme est tiré et pétri, qui appelle celui du potier (Eccli. 33, 13; Is. 43, 9, etc.) et du vase fragile que sont les hommes ( 2 Cor. 4,7 ); celui, d'autre part, du reddite Caesari évangélique (Mat. 21, 22) et de l'image et ressemblance divine imprimée en l'homme lors de sa création. Les deux symbolismes paraissent dans une scène en trois tableaux, disposés sur l'une des montées de l'arche de la sagesse, et qui scandent les étapes (cognitio, meditatio, contemplatio) de la guérison de l'ignorance que comporte le péché d'origine. Cognitio et meditatio sont les premières étapes de la restauration du vase brisé, dont les fragments sont recueillis pour être à nouveau rassemblés, puis, la contemplatio intervenant, le feu de l'amour les liquéfie pour que la similitudo soit restaurée. Ce glissement d'un thème à l'autre, et la substitution curieuse de la terre modelée au métal liquéfié, est moins incohérent qu'il n'y paraît: la similitudo finale renvoie à la similitude originelle qui s'accompagnait de l'intégrité de l'âme et à l'opus conditionis; le vase porté au sortir de la caverne est donc à identifier à l'âme

17. Voir chez Hugues par exemple De arrha animae, éd. P. Sicard (tr. éd.) et Dominique Poirel, Turnhout, Brepols, («Sous la règle de saint Augustin», 3; «L'œuvre de Hugues de Saint-Victor», 1), 1997, p. 281-283, 1. 844-865: «Ma conscience est ensoleillée, j'oublie la peine de toutes mes misères passées, mon esprit exulte, mon intelligence s'éclaire, mon cœur s'illumine. [...] Il y a à l'intérieur quelque chose que mon amour tient embrassé, et je ne sais ce que c'est, et cependant je fais tous mes efforts pour le retenir et ne le perdre à jamais. Mon esprit lutte en quelque sorte délicieusement pour ne pas se retirer de celui qu'il veut étreindre toujours [...] - En vérité c'est lui ton bien-aimé qui te visite. Mais il vient insaisissable. Il vient pour te toucher, non pour que tu le voies; pour t'avertir, non pour que tu le saisisses ». Pareillement, dans le De archa Noe IV, iv, cité plus haut; ou en Bernard, Homélies sur le Cantique 74, 6-7. 
humaine, vase fragile et œuvre du potier, qui aura pu être cuit au four, mais qui, à cause d'une «pierre de scandale», est fragmenté.

Ainsi, outre les connotations majeures que présentait pour le victorin l'idée du vase d'argile - principalement l'évocation de la glèbe originelle de la Genèse - cette substitution pouvait offrir à ses yeux de consonner avec une représentation des suites du péché originel qui, dans l'ouvrage en cause, le De archa, insiste sur la division et la fragmentation de l'homme, consécutive au (ou constitutive du) péché des origines :

Cor ergo hominis, quod prius amori divino affixum stabile perstitit et unum amando unum permansit, postquam per desideria terrena difluere cepit, quasi in tot divisum est, quod ea sunt que concupiscit. Sicque fit, ut mens que verum bonum amare nescit, nunquam valeat esse stabilis, quia desiderii sui finem in his quae amplectitur non inveniens, dum semper se desiderio extendit, sequens quod consequi non valet, nunquam requiescit. Hinc igitur nascitur motus sine stabilitate, labor sine requie, cursus sine perventione, ita ut semper inquietum sit cor nostrum, donec illi adherere ceperit, ubi et desiderio suo nihil deesse gaudeat et ea quae diligit semper mansura confidat ${ }^{18}$.

Et plus proche encore du propos présent:

In calore orientis creatus fuit primus homo, postea superbiendo transivit ad frigus orientis, quasi ad aquilonem ad consortium diaboli. Deinde per concupiscentiam carnis cecidit ad calorem occidentis et per ignorantiam ad frigus occidentis. Sicque per quatuor partes mundi diffusus est et dissipatus. Cum autem colligitur et revocatur, primum de quatuor partibus mundi ad archam, id est ad Ecclesiam, accedit, et inde sursum ascendens paulatim se in unum colligit ${ }^{19}$.

18. De archa Noe, éd. cit., I, i, 46-57: «Alors que l'amour divin qui le liait le maintenait dans la stabilité et qu'il restait unifié par un unique amour, le cœur de l'homme, après avoir commencé à se répandre en désirs terrestres, se trouve divisé à proportion que se multiplient les objets de sa concupiscence. Dès lors, l'esprit qui ne sait pas aimer le bien véritable ne peut jamais être stable, car, ne trouvant pas l'apaisement de son désir dans les choses qu'il embrasse, poursuivant ce qui ne peut être atteint, jamais, dans cette tension permanente vers ce qu'il désire, il ne trouve le repos. De là naissent ce mouvement sans arrêt, ce labeur sans repos, cette course sans fin, en sorte que notre cœur est dans l'inquiétude tant qu'il n'en vient pas à s'attacher à celui en qui il puisse se réjouir de ce que rien ne manque à son désir et avoir l'assurance que ce qu'il aime durera toujours ».

19. Libellus de formatione arche, éd. cit., IV, 202-210. «Le premier homme fut créé dans la chaleur de l'orient; ensuite, dans son orgueil, il passa au froid de l'orient, comme s'il se rendait à l'aquilon, en compagnie du diable. Puis la concupiscence de la chair le fit choir dans la chaleur de l'occident, et l'ignorance tomber dans le froid de l'occident. Et le voici répandu et dissipé dans les quatre parties du monde. Mais quand il est recueilli et rappelé des quatre parties du 


\section{La monnaie, image et ressemblance}

À cette division et fragmentation de l'âme, la méditation remédie partiellement par son activité, avant que la contemplation ne prenne le relais. Intervient alors la substitution de l'imagerie du métal en fusion à celle de la poterie, en des termes qui, exposant les procédés du fondeur médiéval, ne supportent nulle ambiguïté: $a d$ similitudinem fabri conflans, quasi per fistulam, quasi in monetam.

Depuis Tertullien, l'épisode évangélique qui a donné lieu au «Rendez à César ce qui est à César et à Dieu ce qui est à Dieu» (Mat. 21, 22), avait été occasion d'exposer une doctrine de l'image divine: l'image de César (qui in nummo est) et celle de Dieu (qui in homine est). Le reddite qui introduit dans ce qui serait une comparaison statique l'idée du retour à Dieu (ut Caesari pecuniam reddas, Deo, temetipsum $)^{20}$, pouvait également prendre appui sur la parabole évangélique de la drachme perdue et retrouvée (Lc. 15, 8-9). Saint Augustin aura mis en œuvre ces données, dont l'alter Augustinus médiéval que fut Hugues devait s'inspirer:

Signatum est super nos lumen vultus tui, Domine. Facti sumus ad imaginem Dei, nec resculpimur nisi ab illo qui fecit nos, nec reformamur nisi ab illo qui crevit. Ergo Signatum est super nos lumen vultus tui, Domine, ut simus nummus tuus et condamur in thesauro tuo. [...] Haec est moneta Christi : signatus est in nobis vultus imperatoris nostri ${ }^{21}$.

Trois connotations importantes apparaissent chez Augustin. À l'idée du retour-restitution est jointe celle du principe de cette restitution de l'image en l'homme et de la restitution de l'image qu'est l'homme à Dieu. Ce principe de restauration est identique à celui de la création: refait celui qui a fait. La reconstitution est

monde, il accède d'abord à l'arche, c'est-à-dire à l'Église, d'où, en s'élevant, il s'unifie progressivement jusqu'à ce qu'il parvienne au sommet».

20. De idololatria 15, à quoi on peut ajouter De fuga in persecutione 12 («imaginem et monetam ipsius, inscriptam nomine eius, id est hominem christianum»).

21. Aug., Ser. Dolbeau 16, 12 (Augustin d'Hippone, Vingt-six sermons au peuple d'Afrique, éd. François Dolbeau, Paris, Etudes augustiniennes, 1996, p. 128): «Sur nous est empreinte la lumière de ton visage Seigneur (Ps. 4, 7). Nous avons été faits à l'image de Dieu, et personne ne nous refaçonnera, sinon celui qui nous a faits; et personne ne nous remodèlera, sinon celui qui nous a créés. Donc Sur nous est empreinte la lumière de ton visage Seigneur, pour que nous soyons des monnaies frappées à ton effigie. [...]Voici donc la monnaie du Christ: est gravé en nous le visage de notre empereur». 
comme une frappe nouvelle: «pour que soit pour ainsi dire regravée sur la pièce, c'est-à-dire en notre âme, l'image de notre Dieu et que nous soyons réintégrés dans ses trésors $»^{22}$. La gravure peut aussi être restituée par un burinage décapant (resculpatur in nummo ${ }^{23}$ ). Un second élément est celui de la connaissance, car, «en cette image que nous sommes, la ressemblance avec Dieu sera parfaite quand sera parfaite la vision de Dieu $»^{24}$. Troisième remarque enfin: la dimension cognitive, jointe à l'idée de réimpression, de frappe, voire de burinage, semblerait impliquer une activité exercée avec détermination ou quelque acte d'intelligence pure ou une illumination qui s'accommoderait d'être une lumière «froide». Pourtant on note la présence, dans des textes augustiniens parallèles sur l'imago et la moneta, des notions de chaleur, de réchauffement:

Homines ergo, reddentes Deo quae Dei sunt, si Caesari reddunt quod Caesaris est, id est reddentes Caesari imaginem suam, et reddentes Deo imaginem suam, erigunt ipsam mentem suam, non ad se, sed ad artificem suum, et ut ad lumen unde sunt et ad calorem quemdam spiritalem unde fervescunt et unde remoti frigescunt et unde recedentes contenebrantur ${ }^{25}$

Froid et chaleur, retour à Dieu, création première, lumière et connaissance: autant d'amorces pour l'introduction, chez le victorin, en un contexte de liquéfaction où intervient la ferveur de la charité, d'une nette dimension cognoscitive.

22. Deux autres images interviennent dans ces derniers mots qu'elles soustendent: d'abord celle de la drachme perdue et retrouvée (alors l'idée pointe vers la remise en possession de son légitime propriétaire; passe au second plan la déformation puis la refonte de l'effigie portée par la drachme) puis celle, plus lointaine ici, de la brebis perdue, qu'il faut par une quête peineuse, réintégrer au troupeau.

23. En. in Ps. 94, 2, qui reprend, fere ad litteram, les mêmes thèmes: "similitudine ad quam facti sumus, quam in nobis peccando corrumperamus, quam peccatorum remissione recepimus, quae in nobis renovatur intus in mente, ut tamquam resculpatur in nummo, id est in anima nostra imago Dei nostri, et redeamus ad thesauros eius».

24. De Trinitate, 14, 17, 23 ( CCSL», 50), p. 455, cité par Marie-François Berrouard, Saint Augustin, Homélies sur l'évangile de saint Jean, Paris, Études augustiniennes, («Bibliothèque augustinienne», 73A), 1988, p. 486-487, note complémentaire 20 «Nous sommes la monnaie de Dieu».

25. En. in Ps. 103, s. 4, 2, 28-35 («CCSL», 40): «Les hommes, rendant ainsi à Dieu ce qui est à Dieu et à César ce qui est à César, c'est-à-dire rendant à César son image et rendant à Dieu son image, élèvent leur esprit, non vers eux-mêmes, mais vers leur artisan suprême et vers la lumière d'où ils proviennent, et vers cette chaleur spirituelle par laquelle ils sont en ardeur ou se refroidissent quand ils en sont loin, s'enténèbrent quand ils s'en éloignent». 
Le vase que porte le personnage au sortir de la caverne est l'âme rationnelle, dans son intégrité: «l'âme rationnelle, dans sa santé, est un vase solide et intègre, sans nulle corruption $»^{26}$. Cette intégrité consiste dans la vérité et dans la bonté; dans la vérité quand l'âme connaît, dans la vérité quand elle aime. La connaissance est corrompue par l'ignorance qui insère en elle quelque ferment mauvais; et l'amour, corrompu par la convoitise, ôte la santé: «la clarté de la connaissance est comme une certaine beauté de l'âme; la sincérité de la dilectio en est la santé» (De oratione dominica). L'ignorance est progressivement guérie par la lectio, puis par la meditatio et parachevée par la contemplation qu'accompagne la liquefactio. Cette séquence confère ainsi à la liquéfaction un aspect nécessaire à l'accomplissement entier de l'opus restaurationis; c'est également signifier, en même temps qu'une continuité, un décrochement et changement d'ordre opéré par l'entrée dans l'expérience mystique.

Une particularité paraît pour la première fois dans la littérature médiévale unie à l'image du fer en fusion: si la liquéfaction avait pour sujet d'inhésion l'âme (anima), les facultés évoquées à ce propos étaient presque toujours la volonté ou affectivité, avec l'amor, et de façon indirecte la mémoire, avec le ressouvenir des preuves de l'amour. Paraît ici la connaissance. L'amour est encore présent et opère la liquéfaction (per ignem divini amoris liquefaciendo), mais la connaissance est ce en quoi s'opère cette montée (ascensus) qui est in cogitationibus (VII, 24): il faut restaurer l'intégrité de l'âme humaine et c'est sur les débris épars de cette intégrité brisée par l'ignorance qu'agit la connaissance, en les rapprochant par la méditation et, plus excellemment, par la contemplation. Celle-ci opère, grâce à la fusion, la réunification des fragments de l'âme et la restitution de la connaissance originelle. La connaissance connaît; l'amour liquéfie; la liquéfaction permet la connaissance contemplative et celle-ci est uniment restauration de l'image divine.

La notion de liquéfaction dans ce passage majeur permet donc au victorin une double intégration: celle du symbolisme de la monnaie et de l'image de Dieu à celle du métal en fusion, d'une part, et, d'autre part, l'introduction du symbolisme du vase d'argile dans celui du métal liquéfié. On notera la substitution à la «frappe»ou 
au «décapage», principalement exploités par la période patristique pour la pièce à l'effigie de César, de l'idée d'une refonte, à peine esquissée dans les périodes antérieures.

\section{Course, suite et fuite: les choses de la physique et les mots de la mystique}

Très nettement chez le victorin, la liquéfaction précède et rend possible un dynamisme: celui de la sequela Christi. La «course» qui dans un contexte de mystique sponsale prenait la forme d'une course à la poursuite du Bien-aimé, devient dans une mystique de l'image et ressemblance, la course du métal en fusion dans le canal qui l'achemine vers la matrice où une refonte doit être faite. L'insistance est ici également sur l'idée de vitesse, d'entraînement naturel, qui atténue ou fait disparaître l'effort de la montée: si la méditation emprunte parfois des chemins sinueux et revient sur ses pas, la contemplation est «uniforme et simple». Aussi estelle figurée par la rectitude d'un trait de couleur, qui représente le conduit par où s'écoule le métal en fusion, sans méandres et selon les voies les plus directes et toute la vitesse dont il est susceptible, libre de tout ralentissement d'ordre externe ${ }^{27}$. Il est à noter également que le «naturalisme» de la représentation qui use du vocabulaire technique de la fonderie, ne va pas jusqu'à inverser une direction: l'écoulement qu'amène la liquefactio et qui se fait normalement de haut en bas reçoit de la maquette de l'arche où les trois personnages sont introduits, une direction qui le fait à la fois monter de bas en haut et progresser des parois extérieures de l'arche vers son centre, qui est en même temps son sommet.

La prise en compte des éléments physiques et de leur cours naturel qui sert au langage mystique supporte, au moment même où la considération morale le cède à la considération mystique, une inversion du cours naturel des éléments primordiaux. L'eau, expliquera le même traité, peut représenter «la convoitise de ce monde» qui, «à la ressemblance de l'eau, court vers le bas et tend toujours vers le fond $»^{28}$. Rendu liquide par le feu, le métal de l'âme

27. Libellus de formatione arche, éd. cit., IV, 127-129: «Hic autem versus distinctionem non habet, sed unus ductus coloris ab imo sursum trahitur, quia contemplatio uniformis est et simplex ».

28. De archa Noe, éd. cit., IV, vii, 14-17 (cité par D. Poirel, «L'eau et le feu 
ne tendra pas vers le bas, mais vers le haut : c'est que le plus précieux des quatre éléments, le feu, lui communique avec la condition liquide, une de ses propriétés, qui est la motion et la motion vers le haut. Dans les quatre éléments de ce monde, «le feu [...] ne peut [...] être conservé dans l'immobilité, en sorte qu'il ne bouge point, [...] le feu est seul à ne pouvoir ni être contenu, ni même être mis en mouvement de l'extérieur, puisque tout mouvement est en lui et vient de lui ${ }^{29}$. Et sa place dans la hiérarchie et la localisation des quatre éléments est la plus élevée. Ce à quoi est communiqué cette qualité élevante est précisément ce qui est le plus bas et dont la propriété est d'être de soi immobile, à savoir un métal, qui pour être éventuellement précieux, n'appartient pas moins à l'élément terre. Ce qui vaut plus encore des deux étapes premières de la restauration, où les fragments du vase brisé font référence immédiate à la glèbe originelle.

Les gloses et amplifications victorines supposent la classification de la physique ancienne des quatre éléments en deux éléments actifs, le feu et l'air, et deux éléments passifs, la terre et l'eau: on tient là également un trait qui pouvait recommander la métaphore du feu et du fer, plus encore que celle de la lumière dans l'air ou de la goutte dans l'océan. La métaphore du feu et du fer en effet, à la différence des deux autres, se prête à exprimer un des traits essentiels de l'expérience mystique, à savoir du côté du sujet, ce Hierotée, patiens divina, la passivité et la réceptivité : car pour la physique ancienne, si «le fer est un corps, [...] la chaleur n'est pas un corps, mais un pâtir $»^{30}$. Enfin, on notera que trois des quatre éléments sont ainsi réunis : la terre à travers le métal, l'eau, à travers la condition désormais liquide du métal, et le feu, instrument du transitus sans transformation que subit le métal liquéfiée ${ }^{31}$.

chez Hugues de Saint-Victor (†1141)», dans Gérard Capdeville (éd.), L'eau et le feu dans les religions antiques, Paris, De Boccard, 2004, p. 349-370, p. 354.

29. De unione corporis et spiritus (PL 177, col. 286AC), cité par D. Poirel, art. cit., p. 351.

30. J. Pépin, art. cit., p. 353, qui fournit également des précisions sur les intermédiaires patristiques entre la physique grecque et les utilisations médiévales de cette triple comparaison «longuement analysée par Aristote, reprise dans le stoïcisme, transposée par les auteurs chrétiens au problème de l'union à Dieu » (p. 373) et utilisée également pour les doctrines christologiques ou eucharistiques.

31. L'air ne paraît pas : il sert plutôt à la métaphore de la lumière qui le traverse et l'illumine. 
Cet usage des réalités physiques était induit par les origines, bibliques chez nos auteurs, du terme «liquéfaction», associé, on l'a vu à la terre (liquefacta est terra, Ps. 74, 4), ou à la cire (sicut cera liquescens, ou liquefacta, Ps. 21, 15), ou aux cieux (caeli sicut fumus liquescent, Is. 51, 6). La première occurrence induisait la possibilité d'introduire également le thème des saisons de l'âme, variation de celui des «âges de la vie spirituelle»: à l'hiver où la terre est gelée, constricte et indurée, succède, grâce aux rayons du soleil printanier, une période qui voit, avec l'amollissement du sol, la floraison. Le Cantique des cantiques (Iam hyemps transiit, imber abiit et recessit, II, 11) pourra être le lieu privilégié de tels développements :

Quia igitur hiemps transiit, flores apparuerunt, et quia imber abiit et recessit vinee florentes odorem dederunt. Hiemps transiit, flores apparuerunt, nam ubi gelu brume verno calore liquatum solvitur continuo terra radiis tepentibus tacta ad partum aperitur ${ }^{32}$.

Lieu d'autant plus privilégié que la simple mention de la «terre» jointe à celle de la «liquéfaction» attire à soi une constellation de termes du vocabulaire mystique ou de thèmes spirituels: jardin mystique; floraison et fructification; rayons du soleil de la contemplation; amollissement ou endurcissement; exercice des sens spirituels, en particulier celui de l'odorat (on sent les fleurs) - un des moins attestés dans la littérature spirituelle médiévale comparativement au toucher - et du goût (on goûte le fruit qu'ont donné les fleurs).

\section{LA LIQUÉFACTION BERNARDINE : A SEMETIPSA LIQUESCERE}

Saint Bernard s'est arrêté dans son commentaire du Cantique bien avant le verset fameux du c. 5, qui, à n'en pas douter, lui aurait fourni occasion de développements multiples. La même

32. Hugues de Saint-Victor, Pro assumptione virginis, éd. P. Sicard, tr. Bernadette Jollès, Turnhout, Brepols, («Sous la règle de saint Augustin», 3; «L'œuvre de Hugues de Saint-Victor», 2), 2000, p. 139, 1. 350-354: «C'est donc parce que l'hiver est passé que les fleurs ont apparu, et parce que la pluie a cessé et s'en est allée que les vignes en fleur ont livré leur odeur. L'hiver est passé, les fleurs ont apparu, car là où fond ce qui est gelé par l'hiver et qui est liquéfié par la chaleur printanière, immédiatement la terre, touchée par de tièdes rayons, s'ouvre pour produire». 
remarque vaut pour le Commentaire sur le Cantique de Guillaume de Saint-Thierry qui ne dépasse pas le c. 3. Chez cet intime de saint Bernard la seule mention de notre thème, dans son De natura et dignitate amoris, le prend en mauvaise part; il est absent de sa Lettre aux Frères du Mont-Dieu, de son De contemplando Deo, de ses Oraisons méditatives. Il faudra attendre la continuation du commentaire du Cantique par Gilbert de Hoyland pour qu'un usage fréquent, mais peu approfondi, soit fait de la notion. On en peut déjà retirer la notation, paradoxale, que la liquéfaction mystique n'est pas originellement dans le monde cistercien, un corrélat de la mystique sponsale.

Chez Bernard, c'est dans le De diligendo Deo que notre thème fait pour la première fois son apparition ${ }^{33}$. Ce passage fameux a arrêté plusieurs commentateurs modernes ${ }^{34}$. Il avait pu inspirer Ruusbroec, entrer dans l'argumentaire critique de Gerson dans son De mystica theologia contre le mystique flamand et dans celui de ses défenseurs, puis après son utilisation par les spirituels baroques et le mouvement quiétiste, leurs apologètes ou contradicteurs. Nous nous arrêterons à quelques éléments marquants, où quelques traces éventuellement victorines peuvent se déceler sous la réception bernardine mais où elles sont infléchies ${ }^{35}$.

33. Sur la datation relative du De diligendo Deo, voir l'introduction à son édition bilingue («Sources chrétiennes», 393), p. 27-28. Le De gratia et libero arbitrio et le De laude novae militiae sont contemporains de l'opuscule (la liquefactio en est absente); lui sont certainement antérieurs le De gradibus humilitatis, l'Apologie et les quatre Homélies mariales, où ne paraît pas davantage la liquefactio.

34. Voir, entre les plus fameux, Étienne Gilson, La théologie mystique de saint Bernard, Paris, Vrin, 1947, p. 144-146; J. Pépin, art. cit., p. 337-344.

35. Bernard a-t-il eu comme inspirateur direct de la métaphore le De quatuor gradibus violentae caritatis de Richard de Saint-Victor, qui nous arrêtera plus loin? Jean Pépin (art. cit., p. 340) voyait pour sa part, au contraire, en Bernard «la source, directe ou indirecte, unique ou partielle [...] de Richard de Saint-Victor». Les progrès dans la datation relative des œuvres tant de Richard que de Bernard ou de Hugues n'appuient pas cette position ou en suggèrent une autre. Le De quattuor gradibus paraît bien un texte de la maturité de Richard, une composition du temps de son priorat à l'abbaye parisienne, ce qui le place «après 1162 » et après la rédaction de ses deux traités du Benjamin maior ou minor et bien après le $D e$ diligendo Deo, rédigé entre 1132 et 1135 . Richard ne pourrait donc être, à s'en tenir aux seules relations littéraires, l'inspirateur de Bernard pour la métaphore du fer en fusion. Le victorin fut-il inspiré par Bernard? Il nous paraît plus probable que Richard a reçu le thème en milieu victorin, et du De archa Noe (1126-1127) de Hugues de Saint-Victor comme de son Libellus de formatione arche (1128-1129) qui, pour le premier, mentionne la liquéfaction ou, pour le second, on l'a vu, en développe au long la figuration imagée. En outre, ces deux œuvres hugoniennes 
Le thème de l'image et ressemblance que comporte l'utilisation de la pièce de monnaie paraît au début du passage dans l'évocation d'une loi nécessaire de la vie spirituelle: «il arrivera assurément qu' un jour l'œuvre se conforme à son auteur et s' accorde (conformet et concordet) à lui », c'est-à-dire que la volonté humaine soit si accordée et conformée à la volonté divine qui a tout fait pour lui, que la première ne désire plus que l'accomplissement de cette pure volonté en sorte que dans son affection «il ne se mêle plus rien qui lui soit propre». L'image et ressemblance restituée est ici, comme dans la majeure partie de la tradition, dans la ligne de l'amour et de l'affectio ${ }^{36}$.

Cette désappropriation, cette désoccupation et ce détachement qu'implique la libération du proprium, note l'insertion explicite, sous une de ses modalités, du thème de «l'anéantissement» qui en milieu bernardin est rendu par le verbe deficere. Très révélatrice est l'expression a semetipsa liquescere. Ignorée de Hugues avant Bernard, mais employée par Richard après Bernard, elle connote, plus que le simple liquescere, la notion d'anéantissement et de defectio, qui prend ici le sens d'une défaillance de l'amour-propre en lequel l'affectio y étant engagée trouvait sa consistance propre. En sorte que le a semetipsa évoque la dureté qui était le partage de

furent diffusées largement et précocement en milieu cistercien: le victorin a pu prendre part, comme maillon et chaînon, à la transmission du thème du «fer liquide». Nous inclinerions cependant à considérer avec Jean Pépin qu'il est peu vraisemblable que les éléments de la triple comparaison du fer, de la goutte et de l'air, aient été réunis par le Cistercien en étant empruntés à des sources différentes, et Bernard serait plutôt «redevable de l'ensemble à un seul auteur». Si toutefois des emplois séparés des trois métaphores ont pu jouer un rôle dans leur adoption par le Cistercien, il faudrait mettre Hugues de Saint-Victor au rang de ceux qui en furent les chaînons transmetteurs. L'acclimatation et l'accréditation en milieu scolaire de cette notion si peu connaturelle au monde des écoles sont largement redevables au victorin et à l'autorité qui lui était reconnue.

36. La position de Hugues de Saint-Victor, qui donne une place première à la connaissance, n'est pas un hapax: le cistercien Gilbert de Hoyland, commentant le Cantique ( $P L$ 184, col. 233CD, cité par R. Javelet, op. cit., p. 430), distingue l'échauffement qui vient de la flamme de l'amour, de la liquéfaction qui fait voir: «Cognata sunt sibi invicem liquidum et calidum. In his duobus contemplationis usus consistit. Quod liquidum est, calorem promptius concipit et conceptus calor vice versa quod liquidum reperit, liquidius reddit. Quod dico calidum et liquidum hoc est, ac si aliis dicatur nominibus, succensum et sincerum. Calidum est, eo quod amat; sincerum et liquidum, quia quamdam amati in se speciem repraesentat. Calidum, quia ardet; liquidum, quia videt. Calidum, quia inflammatur; liquidum quia dilecti imagine informatur». 
l'affectio agrippée à sa convoitise au point de lui être identifiée. La defectio accompagne la liquefactio comme un de ses effets et est conditionné par elle comme sa cause.

Le mouvement impulsé n'est plus une course à la suite du Bienaimé, mais un passage et écoulement de l'affectio dans la volonté divine: «l'attachement humain se liquéfie et se déverse totalement dans la volonté de Dieu» (a semetipsa liquescere, atque in Dei penitus transfundi voluntatem), lequel a voulu «être tout en tous», ce qui demande que ne subsiste plus «dans l'homme quelque chose de l'homme», à savoir une volonté qui ne serait plus conforme au vouloir divin par son attachement au proprium, lequel disparaît quand la volonté ne se veut plus que comme voulue par Dieu et permettant ainsi «l'accomplissement de sa volonté en nous». Le victorin allait jusqu'à la coulée du métal dans la matrice, Bernard s'arrête sur cet écoulement, laissant la pensée concevoir comme un état de passage permanent.

Enfin, cette affectio de la volonté est ainsi transmuée parce que divinum est quod sentitur: on a alors la phrase lapidaire promise à la célébrité: sic affici deificari est. On pourrait rendre le sic affici par «être ainsi touché »" ${ }^{37}$, en entendant «touché» au sens d'être ému ou affecté, mais sans exclure l'exercice du sens spirituel du toucher intérieur: touché divinement par le vouloir divin, l'affectus ne peut plus que ressentir le seul divin, à savoir la volonté divine et s'en trouve «déifié» à savoir conformé à elle, et s'écoule en elle.

\section{Dilatation, défaillance et bouillonnement : LES ACCOMPAGNEMENTS DE LA LIQUÉFACTION CHEZ LES MOINES ET LES CHANOINES}

Si la liquéfaction paraît appartenir au nucleus de l'expérience mystique, trois autres phénomènes sont souvent présentés dans un rapport étroit avec cette liquéfaction: la dilatatio qui est montrée comme un effet immédiat; la défaillance, tantôt comme une de ses suites immédiates ou de ses conditions ultimes, ou encore comme un simple aspect, dans celui qui expérimente la liquéfaction, de celle-ci; le bouillonnement, enfin, à nouveau comme une suite 
prochaine de la liquéfaction et comme la cause immédiate de l'excessus.

\section{La liquéfaction et les directions de la dilatation ${ }^{38}$}

L'écoulement que déterminait la liquéfaction bernardine était un «déversement dans la volonté de Dieu», celui de la liquéfaction hugonienne était une ascension vers la matrice originelle: les connotations sont toujours vers le haut. Mais d'autres effets de la liquéfaction suggèrent la notion d'horizontalité, voire de descente. «Le cœur liquéfié se dilate», mais cette dilatation se fait, pour l'auteur de l'Épître à Séverin sur la charité, horizontalement ${ }^{39}$ :

Emolitum autem cor [...] dilatatur in caritate, a dextris et a sinistris usquequaque diffunditur, subveniens pariter hostibus et amicis, atque grato quodam spontaneo cursu omnibus occurit ${ }^{40}$.

Cette extension par nappes qui rejoint autrui, quelle que soit sa condition spirituelle ou son attitude à l'égard du mystique, est une

38. La dilatatio cordis est un autre thème du vocabulaire spirituel du XII ${ }^{\mathrm{e}}$ siècle, qui mériterait une étude pour soi-même. Chez Richard de Saint-Victor, la dilatatio est un des modes selon lesquels s'exercent les quatre premiers genres de contemplation (De contemplatione $\mathrm{V}, 2$ ). Une telle dilatatio mentis «fruit de l'industrie humaine et au terme de l'effort méditatif, n'a de soi rien d'extraordinaire». Elle diffère donc des deux autres modes ou états où se trouve l'esprit dans ces genres; la sublevatio connaît le concours de l'effort humain, simple condition de docilité nécessaire, et de la grâce principalement agissante; l'alienatio enfin ne se produit que sous la seule action de Dieu, sans nulle préparation ou disposition. Ces trois modes de contemplation (dilatatio, sublevatio, excessus) sont autant d'étapes nécessaires: l'âme descendue au-dessous d'elle-même (c'est la dilatatio mentis, dont méditation et connaissance de soi sont étapes essentielles, mais où l'on part du sensible, inférieur à l'homme), s'élève ensuite au-dessus d'elle-même (sublevatio mentis) pour être introduite dans la contemplation proprement dite par cette alienatio mentis qui la fait sortir hors d'elle-même dans l'excessus.» (P. Sicard, «Figure Vittorine: Riccardo, Acardo e Tommaso », dans Figure del Pensiero medievale, t. 2, La Fioritura della Dialettica, X-XII secolo, Milan, Jaca Book, 2008, p. 459-537). Une telle dilatatio-mode est donc bien diverse de la dilatatio qui accompagne la liquefactio.

39. «Res liquescendo dilatatur...», «Emolitum cor dilatatur»: Ives, Épître à Séverin sur la charité, éd. cit., p. 9-87, et pour les deux passages cités, n 33, 34. Tissé d'emprunts à saint Bernard, mais proche également de Richard de SaintVictor, auquel il fut longtemps attribué, l'opuscule est d'un certain «Yves», soit cistercien écrivant à Séverin, «sous-prieur de Saint-Denis», soit plutôt moine bénédictin (voir G. Dumeige, pour la critique d'attribution, p. 20-25).

40. Épitre à Séverin, éd. cit., ${ }^{\circ}$ 34: «Le cœur rendu moins dur se dilate de tous côtés dans la charité... Il se répand partout à droite et à gauche, secourant également ennemis et amis, allant au-devant de tous dans une sorte d'avance charmante et spontanée». 
façon de se faire réellement «tout à tous», «se réjouissant de ses progrès, compatissant de ses défauts ». Surtout, c'est un écoulement permanent car il n'implique pas l'épuisement (fluit igitur, sed non effluit), car comme pour le passage et l'écoulement en Dieu, il n'est pas perte, usure ou épuisement de la substance propre. Ce trait servira également, nous le verrons, sinon à situer l'écoulement du liquide par rapport au raptus ou à l'excessus - termes médiévaux pour l'extase - du moins surtout à rectifier les représentations que pourraient s'en faire les lecteurs.

Chez le quasi-contemporain d'Yves, Richard de Saint-Victor (De quatuor gradibus violentae caritatis), on assiste à une kénose, car cet écoulement se fait vers le bas, et à son propos c'est le semetipsum exinanivit paulinien (Phil. 2, 7) qui est explicitement et longuement évoqué:

Sicut metallum liquefactum quocumque ei via aperitur facile ad inferiora currendo delabitur, sic anima in hoc esse ad omnem obedientiam se sponte humiliat et ad omnem humilitatem iuxta divine dispositionis ordinem libenter inclinat ${ }^{41}$.

Cette ductilité entière qu'emporte la liquéfaction fait que le spirituel qui, selon Richard de Saint-Victor, a reconquis un pouvoir perdu sur soi-même et l'ensemble de l'activité humaine (regia potestas), reprend «possession du royaume intérieur (Lc. 17, 21) perdu à la suite du péché par lequel l'homme avait été exilé du jardin du paradis qu'il possédait» (De eruditione I, 1, 48; De statu $\mathrm{I}, 16)$ et «se retrouve pleinement libre dans une indépendance souveraine car, se complaisant dans le seul Bien et dans les seuls vouloirs divins, plus rien ne peut le faire souffrir ni éprouver de contrariétés: tout est ordonné par Dieu et perçu comme tel $»^{42}$.

Chez Hugues de Saint-Victor commentant la Hiérarchie céleste du pseudo-Denys, l'écoulement déterminé par la liquéfaction rend le métal pénétrant (liquidum penetrans omnia) et donne à l'âme de rechercher l'Époux (quaesivit illum quia liquefacta est ad illum)

41. De quatuor gradibus violentae caritatis, éd. G. Dumeige, Paris, Vrin, 1955, p. 126-177, $\mathrm{n}^{\circ}$ 42. «De même que le métal en fusion partout où on lui laisse un passage, s'écoule rapidement et aisément vers le bas, ainsi l'âme se plie facilement à tout signe de la volonté de Dieu [...] toute sa volonté prend la forme exacte de son bon plaisir». Richard parle ici du quatrième degré de l'amour violent, où s'unissent défaillance venant de la ductilité et liquéfaction; pareille association se trouvait déjà au troisième degré, qui connaissait la liquéfaction.

42. P. Sicard, op. cit. (voir n. 38), p. 488-489. 
mais dans une quête ordonnée à s'achever en lui puisque «ce qui est liquide pénètre, et n'a de cesse qu'il n'ait atteint le plus intime» et qu'il appartient à la dilection liquéfiante de «tout pénétrer» (omnia penetrare dilectio). Ainsi l'écoulement se fait ad dilectum, immo potius in dilectum. Ces entrées sont réciproques et mutuelles entre Dieu et l'âme, mais sous l'initiative divine:

Ergo ipse ad te intrabit ut tu ingrediaris ad ipsum. Tunc enim tu intras ad ipsum, quando ipse ad te ingreditur. Quando amor illius cor tuum intrat et penetrat $[\ldots]$ tunc intrat in te ipse et tu quoque intras teipsum, ut ingrediaris ad ipsum ${ }^{43}$.

\section{La défaillance et la liquéfaction}

L'association de la liquéfaction à l'anéantissement mystique et le glissement de l'un à l'autre a pu être fortement suggéré par l'impression du fameux desiderium dissolvi et esse cum Christo paulinien (Phil. 1, 23), qui compte au rang des adages prégnants auxquels s'arrêtent volontiers les spirituels et où surtout, la «dissolution» appelait une image «liquide» ou «liquéfiante». Pareille association ne pouvait qu'être encouragée par la lecture anagogique des psaumes: Defecit caro mea et cor meum (Ps. 72, 26). La defectio porte sur le «cœur», tout comme la liquefactio, ce qui amènera leur rapprochement naturel: «son âme est liquéfiée (liquefacta est) au feu divin et à la flamme du désir, au point de pouvoir dire avec le prophète: Ma chair et mon caur ont défailli» ${ }^{44}$.

Ce que recouvre cette défaillance-anéantissement est explicité par Richard qui précise à cette occasion ce qu'implique pour les puissances (volonté propre et libre arbitre) cette ductilité entière qu'amène la liquéfaction:

In hoc statu $[\ldots]$ anima in illum quem diligit tota liquescit $[\ldots]$. Quemadmodum igitur in liquidis vel in liquefactis nichil duritie, nichil

43. Expositio in Hierarchiam Caelestem, PL 75, col. 1038B : «Tu entres en lui lorsqu'il entre en toi. Quand son amour entre en ton cœur et le pénètre [...] alors il entre lui-même en toi pour que tu rentres en toi-même afin de faire ton entrée en lui ». Sur la pénétration naturelle du liquide, la formulation incisive, en forme d'adage physique ou métaphysique, d'un milieu proche de l'abbaye de Vercelli est à retenir: "Omne calidum et liquidum est penetrativum» (éd. Jeanne Barbet et Francis Ruello, Un commentaire vercellien du cantique des cantiques: Deiformis anime gemitus, Turnhout, Brepols, («Sous la règle de saint Augustin», 10), 2005, V, p. 370, 1. 418-419.

44. Aelred de Rievauld, Sermon 73, 9 («CCCM», 2B). 
firmitatis videtur inesse [...], et quemadmodum in languidis videmus nichil proprii vigoris, nichil native virtutis habere, sed totum quod vivat ex alieno arbitrio pendere, sic qui ad hunc tertium amoris gradum profecerunt, nichil iam propria voluntate agunt, nichil omnino suo arbitrio relinquunt, sed divine dispositioni omnia commitunt, omne eorum votum, omne desiderium ad divinum pendet nutum, ad divinum spectat arbitrium ${ }^{45}$.

Source importante d'inspiration de cette dernière vue, encore un psaume (cum defecerit virtus mea, Ps. 70, 9): la defectio pourra donc s'appliquer à la défaillance des puissances natives (virtutes), non seulement quand elles s'en remettent à des puissances supérieures en lesquelles elles défaillent et qui les recueillent, mais en particulier quand elles touchent au bout de leurs possibilités et se heurtent au transitus vers un état ou passage à un degré spirituel où il ne leur est pas donné de faire pénétrer. La defectio alors prélude au relèvement par intervention d'une autre instance ou par initiative divine nouvelle ${ }^{46}$. Ici encore le latin biblique aura fourni ses termes au glossaire mystique.

45. Richard de Saint-Victor, De quatuor gradibus violentae caritatis, éd. cit., $\mathrm{n}^{\circ} 41$. «En cet état, l'âme se fond toute entière en celui qu'elle aime [...]. Et comme dans les liquides ou dans les corps liquéfiés, aucune dureté, aucune consistance ne demeure, $[\ldots]$ de même nous ne retrouvons en ces âmes languissantes aucune vigueur propre, aucune force innée; tout ce qui vit en elles dépend au contraire d'une autre volonté [...]. Elles ne font plus rien par leur volonté propre, ne laissent rien à leur décision, remettent toutes choses aux dispositions divines. Tous leurs souhaits, tous leurs désirs sont suspendus aux ordres de Dieu, ils ne considèrent que le bon plaisir divin».

46. On pouvait s'attendre à trouver des exemples nombreux chez Richard de Saint-Victor dont le talent classificateur devait les lui faire multiplier, surtout dans ses deux «Benjamins». Exemplaire est une formulation comme: «ubi humana ratio deficit, ibi Rachel moritur, ut Benjamin oriatur», De duodecim patriarchis, 57, 11-12 («Sources chrétiennes», 419). Défection et «passage» (transire, transcendere) sont un couple constamment attesté, avec ou sans référence explicite à la liquéfaction. On peut en recenser les exemples dans l'œuvre du théoricien de la mystique: «Idem itaque animi affectus circa eamdem rem crescit atque decrescit, et modo vehementer intumescit, et modo pene, modo penitus deficit, et post plenum saepe defectum penitus reviviscit» (De contemplatione 3, 22, $P L$ 146, col. 131D); "Dum mens humana semper ad altiora crescit, dum diu crescendo tandem aliquando humanae capacitatis metas transcendit, fit demum ut a semetipsa penitus deficiat, et in supermundanum quemdam transformata affectum, tota supra semetipsam eat» (ibid. 5, 9, col. 178D); «Cur non et recte dicatur spiritus semetipsum non habere, quando incipit a semetipso deficere, et a suo esse in supermundanum quemdam, et vere plus quam humanum statum transire, et mirabili transfiguratione spiritus ille ab humano videatur in divinum deficere » (ibid. 5, 12, col. 182C), etc. 
Chez saint Bernard (De diligendo Deo, X, 27), qui cite le psaume 72, 26 (Defecit caro mea et cor meum) ${ }^{47}$, deficere paraît exprimer, non tant une suite ou une condition antécédente ou concomitante de la liquéfaction, que cette liquéfaction même vue du côté du sujet, qui «se perd en quelque sorte [...] [n'ayant] plus aucune conscience de soi-même (et omnino non sentire teipsum)». La cessation de la perception de soi, du proprium sera-t-il expliqué plus loin, à quoi s'oppose un écoulement rectiligne contraire à tout retour sur soi et à tout mouvement réflexe, est donc vécue comme une «défaillance». C'est de cette perception centrée sur le sujet que le sujet même est libéré dans la liquéfaction (liquescere a semetipsa ou deficere a semetipsa). L'auteur de l'Épître à Séverin semble aussi équivaloir liquéfaction et deficere, de telle sorte cependant que le second est un aspect de la première et exprime surtout l'abandon (par arrachement, lui-même effet d'une attraction) de «modes» d'agir, d'être, voire de se percevoir: le cœur de celui qui aime doit être liquéfié (a se liquefieri), pour que puissent se réaliser l'assimilation (transformari) et l'écoulement (transfundi), de même qu'une goutte d'eau dans du vin deficere a se tota videtur. Ainsi, celui qui est de la sorte defectus (en fait profectus) «ne sent plus rien de soi-même en soi » ${ }^{48}$. Le De quatuor gradibus de Richard décrit les étapes préparatoires de la liquéfaction du morceau de fer (le fer s'échauffe; le fer rougit) et le terme (le fer fond): et c'est en cette étape qu'il «abandonne totalement son premier état» $(a$ priori statu penitus deficit $\left.{ }^{49}\right)$. La liquidité totale du fer est aussi une defectio.

La défaillance mystique quand elle est mentionnée par nos auteurs dans une proximité de la liquéfaction, ne l'est habituellement qu'au moyen d'un rapprochement qui au plus laisse admettre une

47. Même citation en Guillaume de Saint-Thierry, De natura et dignitate amoris 52. Guillaume connaît (De natura et dignitate amoris 6 ), un sens négatif de la defectio: quand la volonté, simple affectus, est «réduite à elle-même» sans la grâce: alors «elle défaille en elle-même». Entendons qu'elle descend au-dessous d'elle-même en devenant un des vices qui l'emplit. Les yeux intérieurs des sens spirituels eux-aussi peuvent connaître une défaillance, quand il leur faut une nouvelle intervention divine pour «voir celui que j'aimerai et aimer celui que je verrai », De contemplando Deo, $\mathrm{n}^{\circ} 5$ («Sources chrétiennes», 61bis). Guillaume comme la plupart des auteurs joue sur le préfixe : perficere/deficere/proficere (voir De natura et dignitate amoris 49$)$.

48. «De se in se nihil sentiunt» (Épître à Séverin, éd. cit., $\mathrm{n}^{\circ} 32$ ).

49. De quatuor gradibus, éd. cit., $\mathrm{n}^{\circ} 39$. 
concomitance. Un auteur cependant, celui de l'Épître à Séverin sur la Charité, précise le processus de consécution: «par leur vertu et leur audition les cœurs se liquéfient, en se liquéfiant ils défaillent, et en défaillant ils sont rendus parfaits $»^{50}$. La séquence est à comprendre plus comme une hiérarchisation ontologique de réalités concomitantes que comme une succession chronologique. On y repère l'exercice des sens spirituels (auditu), qui amène la liquéfaction du cœur (corda liquescunt), laquelle entraîne la défaillance (liquescendo deficiunt), qui à son tour rend parfait (deficiendo perficiunt). Les deux procédés rhétoriques de la catena et de la gradatio $^{51}$ unis affirment des consécutions causales, mais dont les participes présents laissent deviner qu'on les tient pour instantanées et contemporaines et d'une antériorité ontologique. Le defecit exprime ainsi la transformation vue du côté de l'être du sujet subissant la liquéfaction.

\section{Le bouillonnement et la liquéfaction}

Autre notion souvent, mais non toujours accouplée à la liquéfaction, celle de la bullitio. Saint Bernard avait employé l'image de la marmite sur le feu en contexte mystique:

Moxque ut intus venit, expergefecit dormitantem animam meam; movit et emollivit, et vulneravit cor meum, quoniam durum lapideumque erat, et male sanum [...]. Verum quia haec omnia, ubi abscesserit Verbum, perinde ac si ollae bullienti subtraxeris ignem, quodam illico languore torpentia et frigida iacere incipiunt ${ }^{52}$.

Pour le très ricardien auteur de l'Épître à Séverin, dans le passage cité supra, c'est le cœur liquéfié qui, étant rendu sensible aux paroles du Bien-aimé, bouillonne (sic (...) liquefactum, cor (...) compungitur et ebullit) car il expérimente (experiens illud quod canitur) ce qui est dit au verset fameux du Cantique (anima

50. «Quorum virtute et auditu corda liquescunt, liquescendo deficiunt, et deficiendo perficiuntur» (Épître à Séverin, éd. cit., $\mathrm{n}^{\circ}$ 39).

51. Que Marbode XVII, cité par G. Dumeige (op. cit., p. 39) décrit comme le procédé «où on ne descend pas au mot suivant sans être remonté au précédant».

52. Bernard, In Cant. 74, 6-7 («Sources chrétiennes », 511), p. 168-171: «Sitôt entré, il [le Verbe] a réveillé mon âme endormie, il a remué, amolli et blessé mon cœur qui était dur comme la pierre [...]. Or dès que le Verbe s'est retiré, comme si tu ôtais le feu de dessous une marmite en ébullition, tout cela commence aussitôt à s'engourdir et à refroidir». 
mea liquefacta est). Le bouillonnement paraît dans la suite de la liquefactio, opérée par la voix du Bien-aimé, chez son prédécesseur, Hugues de Saint-Victor:

Quod enim prius acutum fuit et liquidum in dilectione obsistentia alia penetrare valens, superfervidum fit iam, et bulliens in seipso stare non valens. Acutum enim est amoris, cum omnia transeundo despicit, superfervidum autem, cum etiam semetipsum contempnendo relinquit [...]. Fit ergo miro quodammodo ut dum per dilectionis ignem in illum sustollitur qui est supra se, per vim amoris expelli incipiat et exire etiam a se $\mathrm{s}^{53}$

Ce qui fut d'abord pénétrant et liquéfié dans la dilection, devient effervescent (superfervidum) et bouillonnant, incapable de se contenir lui-même (bulliens in seipso), il se quitte lui-même en se méprisant (semetipsum contemnendo relinquit... extra semetipsum projicitur). Cette sortie de soi est un raptus ou excessus où, plutôt que sur la montée vers l'objet, l'accent est mis sur la désertion de soi-même qui suit celle du monde, comme le superfervidum de l'amour qui détache de soi suivait l'acutum de ce même amour par lequel est méprisé tout ce qui est transitoire, et dépassé ce qui passe.

Une dépendance thématique étroite unit Richard à son prédécesseur Hugues, mais le théoricien classificateur qu'est Richard de Saint-Victor dans le De contemplatione $(\mathrm{V}, 7)$ place dans le bouillonnement la cause même du premier mode selon lequel peut se produire l'excessus. Celui-ci en effet peut provenir soit de la grandeur de la devotio, soit de celle de l'admiration, soit de celle de l'exultation. Dans le premier de ces trois modes, l'esprit est élevé au-dessus de lui-même quand la flamme intérieure tire de son premier état l'âme humaine liquéfiée à la manière d'une cire (ad cere similitudinem liquefactam, V, 5). Ce qui introduit à une des deux métaphores filées qui suivent $(\mathrm{V}, 6-7)$ : le premier de ces trois modes est exposé par l'image de la fumée qui s'élève du feu

53. «En effet, ce qui fut d'abord pénétrant et liquide dans la dilection, pouvant pénétrer les autres obstacles, devient effervescent et bouillant sans pouvoir tenir en soi-même. L'amour est, en effet, pénétrant lorsqu'il méprise tout en le dépassant, effervescent quand il s'abandonne soi-même en se méprisant. [...] Il arrive donc d'une certaine manière admirable que, tandis qu'il s'élève en celui qui est au-dessus de lui par le feu de la dilection, il se met à être chassé par la force de l'amour et même à sortir de soi-même» (Hugues de Saint-Victor, In Hierarchiam caelestem, PL 175, col. 1039CD). 
spirituel $(\mathrm{V}, 6)$ ou par celle du liquide que ce feu échauffe dans un récipient:

Incipit liquorem ipsum ab imo evertere, postea vero, nunc in hanc, nunc in illam partem, nunc sursum, nunc deorsum iactare, et paulatim quidem ad superiora attolere [...] tandem autem supra ipsum attolere, et cum violentia quadam interiora evacuare et foras effundere fortiterque eiicere $^{54}$.

Ainsi, continue Richard, de l'esprit de l'homme: enflammé, bouillonnant, irrité contre lui-même, s'indignant vivement contre lui-même, transporté de désir pour les réalités supramondaines et repoussé loin des choses d'en bas et entraîné vers les réalités supérieures par le désir des biens d'en-haut, l'esprit est jeté hors de lui et au-dessus de lui (extra semetipsum et supra semetipsum eiectus), soulevé dans une extase, il est tout entier ravi (in extasi sublevatus totus in superiora rapiatur). La liquefactio produit la bullitio, et celle-ci détermine l'excessus.

\section{DE LA SITUATION DE LA LIQUÉFACTION DANS LE PROCÈS MYSTIQUE MÉDIÉVAL}

Quels que soient les archétypes divers, et pas toujours conciliables entre eux, qu'utilisent nos auteurs pour rendre compte de l'expérience spirituelle (montées, échelles, degrés, genres ou modes, demeures d'une maison, degrés correspondants des vertus, constructions, structures architecturales, fiançailles ou mariage, étapes d'itinéraire, «états» intérieurs successifs), tous s'accordent à situer la liquefactio quand ils en font mention dans les phases terminales du procès mystique. Richard en parle aux troisième et quatrième «degrés de la violente charité»; Bernard l'aborde à l'occasion du quatrième et dernier degré de l'amour «où l'homme s'aime pour Dieu»; Hugues le met en scène par des personnages dans la deuxième moitié de la quatrième des montées successives dont l'ascension rythme la restauration, et qui correspond à une des

54. «Il commence d'abord par faire monter du fond le liquide lui-même; ensuite il le projette tantôt d'un côté, tantôt de l'autre, tantôt en bas, tantôt en haut, et il le fait monter peu à peu jusque tout en haut [...]; finalement il entraîne le liquide au-dessus du récipient lui-même, il le fait sortir sous l'effet d'une sorte de violence interne, le déverse au-dehors et l'expulse avec force» (éd. cit., V, 7, p. 427-429). 
demeures les plus intérieures et élevées de l'âme figurée par les étages de l'arche; Yves en use pour caractériser ce qui est la fin de l'œuvre de l'amour en sa qualité ultime, d'être inséparable et de réaliser l'unition.

Pourtant on trouve mention de la liquéfaction en des états que la classification postérieure classerait dans les états inchoatifs de la vie mystique: ainsi en est-il du toucher-effleurement de l'Époux qui détermine la liquéfaction de l'âme de l'Épouse et sa course. Plus encore, à un degré qui se rapproche davantage du domaine moral que de la mouvance mystique, la liquéfaction assume aussi une tâche de purification des scories morales. On pourrait en conclure, en usant du vocabulaire qui prédominera dans le siècle suivant que, pour les auteurs du XII ${ }^{\mathrm{e}}$ siècle, la notion de liquéfaction est une notion analogique; qu'elle se réalise différemment suivant les états spirituels et que, sans leur être réservée, elle s'emploie préférablement pour les états les plus élevés ou terminaux.

La malléabilité suprême de la liquéfaction n'empêche pas l'existence d'une autre malléabilité, celle qui est obtenue à coups de marteau qui façonnent l'être: chez Bernard (De diligendo Deo IX, 26), poitrine d'airain et cœur de pierre peuvent s'amollir de reconnaissance devant les épreuves dont l'homme fut libéré. Au premier degré de l' amour, un cœur de fer peut devenir, frappé par des châtiments, malléable sous la grâce du libérateur (Épître à Séverin, 20), «une fois échauffé et liquéfié», le cour se laisse «facilement marteler (compungitur) par toute parole du Bien-aimé» (Épître à Séverin, 36), et de citer le Cantique V, 2 (experiens illud quod canitur). Le «martèlement» n'est pas ici une forme, fruste et tout ascétique, antérieure à la liquéfaction mystique, et le compungitur se rendrait mieux par être «frappé» au sens d'être «touché» ou réceptif. La liquéfaction du cœur est alors également ce qui rend accueillant à une parole, éventuellement nouvelle, du Bien-aimé : ce que plusieurs spirituels entendent d'une compréhension renouvelée de passages scripturaires. L'or dont sont faits les chérubins qui couvrent le propitiatoire est rendu ductile également, explique Richard de Saint-Victor (De contemplatione ou Benjamin maior, IV, 7), par le martèlement, quand on choisit de travailler avec un métal repoussé, mais ce martèlement consiste à «obtenir de la bienveillance du Seigneur, par une contrition fréquente du cœur, 
ce à quoi chacun ne peut atteindre par soi-même $»^{55}$. C'est donc une étape, aux mains du vouloir gracié de l'homme, avant la fusion, à laquelle on «ne peut atteindre par soi-même $»^{56}$.

On n'argumente guère la fixation à cette notion d'une place ou d'un rôle précis parmi les phénomènes successifs ou concomitants dont l'ensemble constitue l'état mystique. Lorsqu'une séquence est établie entre ces phénomènes, c'est l'idée simple de causalité et d'engendrement comme linéaire qui la sous-tend le plus souvent. Alors la liquéfaction, effet de l'exercice d'un sens spirituel, est le phénomène engendrant. Peut-être convient-il de n'être pas dupe de ce schéma de présentation. On voit sans doute se grouper autour de la liquefactio les termes de dilatatio, écoulement, excessus, alienatio et leurs équivalents, mais plutôt que de voir dans la liquefactio la cause principale et originelle de chacune de ces composantes, nous inclinerions à voir en cet ensemble, une constellation de termes et de phénomènes, dont il serait vain de chercher le centre organisateur: il suffit de toucher l'un d'entre eux, pour qu'il se transforme en un «centre» temporaire, autour duquel viennent se réorienter les autres élément ${ }^{57}$. Le fait toutefois que la liquefactio soit un de ces termes polarisateurs qu'il suffit de toucher pour qu'il devienne aimantant des autres notions - en attendant que celles-ci le deviennent à leur tour sous l'examen - suffit à confirmer son appartenance, non seulement au lexique de la mystique occidentale, mais à son noyau.

Quant au raptus ou à l'excessus, il est le lieu où se manifeste avec

55. Jean Grosfillier (éd., tr. intr., n.), Richard de Saint-Victor, De contemplatione (Beniamin maior), Turnhout, Brepols, («Sous la règle de saint Augustin», 13; «L'œuvre de Richard de Saint-Victor», 1), 2013, IV, 7, p. 301.

56. Dans les métaphores architecturales si souvent employées au XII ${ }^{\mathrm{e}}$ siècle pour un exposé allégorique ou tropologique, voire anagogique, on recourt également à l'image du tailleur de pierre dont le marteau et les ciseaux sculptent ou du moins taillent les pierres. Notons que la «défaillance» devrait être étudiée en rapport avec une autre notion, très présente dans la mystique victorine, celle de l'alienatio. Il faudrait, pour être complet s'arrêter encore à deux associations que touchent les spirituels médiévaux, et qui sont appelées par les deux conditions du solide et du liquide, de la nourriture et de la boisson. La manne de l'Exode vient au bout de peu de temps à se corrompre: deficere, mais aussi liquescere sont employés à son propos. C'est l'amorce d'une considération sacramentelle dans l'expérience spirituelle. Elle affleure chez Pierre de Celle. En outre, ce passage du solide au liquide fournit l'accroche pour un autre thème mystique, celui du breuvage et de la sobre ivresse (inebriatio).

57. Ce qui suppose en fait que pour les médiévaux, comme pour saint Jean de la Croix, il puisse exister plusieurs centres, selon les divers endroits où l'âme est touchée et qui deviennent alors pour le moment, autant de centres. 
acuité ce qui oblige les auteurs mystiques à recourir si souvent aux oxymores discrets: si la liquéfaction, la malléabilité et la ductilité correspondent de fait à une consistance suprême, elles intéressent l'être profond auquel est rendue sa stabilité foncière, mais auquel également est conférée la possibilité d'entrer dans l'état d'une action, dans un passage, un écoulement, un transitus ${ }^{58}$. On trouve dans l'excessus ou le raptus, mis en rapport avec la liquéfaction, le souci de conserver les deux aspects apparemment contradictoires de la fluidité et de la consistance, de la permanence et de l'écoulement. Un des versets du dossier biblique de la liquéfaction (Sicut cera liquescens in medio ventris mei, Ps. 22, 15) en fournit l'expression qui situe l'écoulement «au centre»:

Fluit igitur [cor], sed non effluit, quia sic se derivat ad alios, ut a se aliquatenus non recedat; et vide tu ne forte propheta senserit id ipsum qui, cum cor suum cere liquescenti comparasset, protinus adiunxit: in medio ventris mei. Liquidum ergo fuit sed extra se non effluxit ${ }^{59}$.

On pourra alors découvrir dans la liquéfaction même l'union à la fois de l'extase et l'enstase du procès mystique, et grâce à elle tenir que, dans l'excessus et le transitus, on est fondé à parler d'être alors à la fois «dans l'esprit et sans l'esprit» (in spiritu (...) et quasi a suo spiritu deficit $)^{60}$ :

Neque etiam necesse est ut in hiis excessibus quibus humani spiritus lutosis divino munere avelluntur, extra se esse dicamus, cum Paulus in suo raptu, an in corpore, an extra corpus fuerit, se dicat prorsus ignorare. Ubi ergo sunt inquis? In eo quod interius est sui. [...] Non ergo educuntur sed inducuntur spiritus in illo interiori cenaculo quodcumque est illud ${ }^{61}$.

58. Et ceci sans se perdre. La persistance de l'être propre est reconnue, mais comme une chose qui va de soi et ne doit faire l'objet que d'une mention peu insistante. Le $\mathrm{XII}^{\mathrm{e}}$ siècle n'a pas partagé au même degré la crainte ultérieure d'une interprétation panthéiste.

59. Épitre à Séverin, éd. cit., n 34: «Le cœur coule, mais sans s’épuiser, car il sait diriger son cours vers autrui sans perdre pourtant rien de soi-même. Vois si ce ne serait pas par hasard l'idée du prophète quand, venant de comparer son cœur à une cire fondante, il s'empressait d'ajouter: au milieu de moi-même. Il s'est liquéfié, mais sans se répandre hors de lui».

60. Richard, De contemplatione, éd. cit., V, 12, 82-83, avec citation révélatrice $\mathrm{du}$ « Defecit in salutare tuum anima mea» du psaume $118,81$.

61. Épître à Séverin, éd. cit., $\mathrm{n}^{\circ} 35$ : «Il n’est même pas nécessaire de dire qu'on est "hors de soi" dans ces sorties de soi-même qui arrachent l'âme humaine à la boue des sens, puisque saint Paul déclare ignorer complètement, quand il fut ravi, "si c'était dans son corps ou hors de son corps". Où sont donc ces âmes, 
C'est situer la liquéfaction au point de création, lieu de passage ascendant et descendant, où l'écoulement d'en haut croise celui d'en bas qu'il aspire, point de création qui devient le lieu de la restauration dans un transitus qui confère une stabilité morale et ontologique, suprême et retrouvée. L'âme va donc per liquidum usque ad solidum, mais non par une étape qui serait désertée pour en atteindre une autre où disparaîtrait la liquéfaction, car:

Immo verius fateor quia colliquescit in solidum amoris Dei et consolidatur in liquidum divinitatis. Totum enim est et liquidum et solidum in deo: liquidum revera quia nos admittit ad viscera misericordiae, solidum quia nos conservat in aeternitate; liquidum quia pius, solidum quia iustus et aeternus; liquidum propter misericordiam, solidum propter iudicium ${ }^{62}$.

\section{Conclusion}

Malgré les avatars de la notion au long de la période médiévale, quelques traits permanents se laissent saisir, que nous présentons, par manière de conclusion et comme matière à une ouverture sur des époques ultérieures.

\section{Agents, sujet}

Le sujet de la liquéfaction ${ }^{63}$ est l'âme (anima). Tantôt il est précisé qu'il s'agit de la volonté (Bernard) ou, en circonscrivant davantage encore, de l'affectio (Guillaume de Saint-Thierry); dans une autre ligne mais qui nous a paru faiblement attestée, la connaissance entre directement en jeu et l'amour est ce qui la promeut (Hugues de Saint-Victor). En d'autres occurrences encore, le lieu de la

demandes-tu? Au plus intime de leur intimité. [...] Ces âmes ne sont pas conduites au dehors, mais introduites dans le cénacle intérieur, quel qu'il soit».

62. Pierre de Celle, De puritate, éd. Jean Leclercq, La spiritualité de Pierre de Celle (1115-1183), Paris, Vrin, («Études de théologie et d'histoire de la spiritualité», 7), 1946, p. 174 (23-26)-175 (1-6): «Pour dire le vrai, l'âme se co-liquéfie dans le solide de l'amour de Dieu, et se co-consolide dans le liquide de la divinité. Car en Dieu, tout est solide et liquide: liquide, car il nous reçoit dans ses entrailles de miséricorde, solide, car il nous conserve dans l'éternité; liquide car il est bon, solide car il est juste et éternel; liquide par la miséricorde, solide par la justice».

63. Hugues parle de subjecta materia pour le feu (Misc. I, 173, PL 177, col. 567B). 
liquéfaction semble être le fond de l'âme ou son centre ou ce que la mystique ultérieure nommera sa «fine pointe». L'agent immédiat est le plus souvent l'amour, ou la dilection ou la charité ou plus précisément l'Esprit saint ${ }^{64}$; quand il est fait appel à la doctrine des sens spirituels, ce sont, sur la base première du Cantique des cantiques, l'ouïe (la voix de l'Époux) et le toucher (l'effleurement de l'Époux, à travers le treillis), ou la vue (l'entrapercevoir à travers le treillis).

\section{Similitude divine}

Semble se vérifier dans l'élaboration de la doctrine de la liquéfaction mystique la loi de la ressemblance restaurée par l'action du semblable ${ }^{65}$ : le fer devient semblable à l'esprit qui l'embrase (Richard). La doctrine spirituelle de l'image et ressemblance est parfois sous-jacente, parfois aussi, mais bien plus rarement, explicite. Cette conformité peut être aussi celle des volontés: la volonté de Dieu a tout fait pour soi; il convient donc que, pour lui être conformée, la volonté humaine veuille cet aspect de la volonté divine, ce qui demande qu'elle soit libérée de tout proprium (Bernard); cette conformité également consiste en l'absolue ductilité que confère à l'âme la liquéfaction: elle épouse, spontanément et en tout et sans résistance aucune, les volontés de Dieu et s'y coule sans heurt ni retard (Richard).

64. L'auteur du De Trinitate insiste sur ce point, voir Richard de SaintVictor, De Trinitate VI, 14, 16-25, éd. Jean Ribaillier, Paris, Vrin, («Textes philosophiques du Moyen Âge»,6), 1958: «Quid enim est Spiritus sanctus nisi ignis divinus? Omnis enim amor est ignis, sed ignis spiritualis. Quod facit ignis corporalis circa ferrum, hoc agit hic ignis de quo loquimur circa cor fedum, frigidum et durum. Nam ad huius ignis immissionem, animus humanus... totus in eius a quo inflammatur similitudinem transit. Nam ex succensione ignis divini incandescit totus, exardescit pariter et eliquescit in amorem Dei». On notera ici encore rapprochés les termes transit et eliquiescit.

65. Elle apparaît ainsi comme le pendant, en anagogie, de la loi de la guérison du semblable par le semblable qui formait, en allégorie, le soubassement de l'élaboration de la théologie sacramentaire en ce même $\mathrm{XII}^{\mathrm{e}}$ siècle: le recours à des éléments sensibles dans les sacrements comme instruments de la grâce médicinale doit guérir l'enlisement dans le sensible, conséquence ou cause de la faute originelle, ou même s'identifiant à elle. Nous avons touché ce point en Théologies victorines, Paris, Parole et silence, 2008, p. 115. 


\section{Effets immédiats}

Une conséquence immédiate de la liquéfaction est la fluidité et la course: course à la suite du Bien-aimé du Cantique qui fuit; ou montée rapide et sans obstacle vers la restauration suprême, ou elle suit la pente de la volonté de Dieu; enfin cette course déterminée par la liquéfaction est un «écoulement» en Dieu ou dans sa volonté, accomplissement du transitus. Peut lui être concomitante, encore que l'insistance ne soit pas toujours sur ce point, une purification, comme par le feu du creuset, des souillures morales, de celles de l'attachement au proprium, ou un amollissement des duretés du cœur, durci contre la volonté divine. Cet aspect négatif du détachement du proprium, de la dureté de cœur, des purifications des souillures morales est exprimé par le verbe deficere, qui peut aussi signifier la défaillance d'une vertu ou puissance humaine parvenue à sa limite extrême; le plus souvent le deficere est le versant négatif et non une condition antécédente de la liquéfaction qui est le versant positif de cette expérience ${ }^{66}$.

\section{Situation}

La liquefactio intervient dans les phases ultimes ou consommées de l'itinéraire mystique; ses rapports avec l'alienatio, la sublevatio ou l'excessus sont rarement explicités, ceux avec la dilatatio ou avec la defectio, plus immédiats, font l'objet de plus fréquentes mentions. Enfin, une doctrine des quatre éléments affleure par places, qui dénonce un soubassement «physique» de la notion et son utilisation dans l'élaboration de la doctrine spirituelle du métal en fusion de trois des quatre éléments primordiaux (eau, terre et feu) et de leurs propriétés naturelles - le chaud et le froid, le solide et le liquide, l'immobilité et le mouvement. Ainsi l'opus restaurationis en ses réalisations éminentes reprend-elle, pour une re-création l'opus conditionis en ses éléments structurels.

S'il faut en croire une recension d'une récente étude ${ }^{67}$, le terme d'anéantissement serait à rattacher «à une tradition médiévale»,

66. Tout comme la «nuit» des mystiques espagnols du XvI ${ }^{\mathrm{e}}$ siècle est l'ombre projetée par la lumière.

67. Lydie Parisse, «La langue de la mystique», recension du volume Pour un vocabulaire mystique au XVII siècle, et en ce recueil, de l'étude d'Hélène Michon (voir n. 1), sur le site en ligne Fabula, la recherche en littérature (Acta), le 7 juillet 2008. 
tandis que «chez François de Sales » celui de liquéfaction «inaugure (...) une mystique moderne». Il nous a semblé que les deux notions étaient, pour le moins, médiévales toutes deux, qu'elles apparaissaient ensemble (sous la forme de la defectio pour l'anéantissement), l'une (la liquéfaction) étant le plus souvent donnée comme cause de l'autre (l'anéantissement): on va donc, inversant la formule déjà mentionnée, «de la liquéfaction à l'anéantissement».

patrice.sicard@irht.cnrs.fr 